\title{
Asymmetric fiber taper for narrow linewidth comb filter
}

\begin{abstract}
The employment of asymmetric optical fiber taper as a means for producing narrow linewidth comb filter is proposed and demonstrated. Fiber taper with different values set for the uptaper and down-taper transition regions was used to produce narrow linewidth comb filter through coupling interaction of light in the asymmetric fiber. Two configurations; single-pass and bidirectional fiber taper filters were studied and analyzed in this project. Results showed narrower 3-dB linewidth for asymmetric taper compared with uniform taper for single pass configuration. The asymmetric taper linewidth was improved further in bidirectional configuration, narrowing down to $2 \mathrm{~nm}$. Bidirectional asymmetric taper filter recorded extinction ratio of $27.14 \mathrm{~dB}$, which was $18 \mathrm{~dB}$ and $3 \mathrm{~dB}$ better than single-pass asymmetric taper and bidirectional uniform taper respectively. The findings emphasize the attractiveness of bidirectional asymmetric taper as a high performance optical filter.
\end{abstract}

Keyword: Asymmetric; Comb filter; Fiber taper; Linewidth 